\title{
The statistical geometry of scale-free random trees
}

\author{
Luca Donetti and Claudio Destri \\ Dipartimento di Fisica G Occhialini, Università di Milano-Bicocca and INFN, \\ Sezione di Milano, Piazza delle Scienze 3, I-20126 Milano, Italy \\ E-mail: Luca.Donetti@mib.infn.it and Claudio.Destri@mib.infn.it
}

Received 11 December 2003

Published 25 May 2004

Online at stacks.iop.org/JPhysA/37/6003

DOI: $10.1088 / 0305-4470 / 37 / 23 / 004$

\begin{abstract}
The properties of random trees (Galton-Watson trees) with scale-free (power-like) probability distribution of coordinations are investigated in the thermodynamic limit. The scaling form of volume probability is found, and the connectivity dimensions are determined and compared with other exponents which describe the growth. The (local) spectral dimension is also determined through the study of the massless limit of the Gaussian model on such trees.
\end{abstract}

PACS numbers: $02.50 .-\mathrm{r}, 05.40 .-\mathrm{a}, 46.65 .+\mathrm{g}$

\section{Introduction and summary}

Graph theory and its applications play an important role in many areas of scientific research, from pure mathematics, to physics, statistics, biology and social sciences. In particular random graphs, that is graphs extracted with some probability from a suitable statistical ensemble, are interesting as a means to implement the intrinsic complexity and/or chaotic nature of many physical, biological and social systems [1].

Among graphs, trees (that is graphs without loops) play a distinguished role: they retain a deep interest and wide applicability while still being amenable to detailed analytic studies. Random trees appear in several distinct contexts, such as polymer physics, critical percolation [2] and two-dimensional quantum gravity (branched polymers [3]).

Generically, in a random graph the local coordination is itself a random variable taking values according to some probability distribution; while in the classical Erdős-Rényi theory of random graphs [4] this distribution is Poissonian, and in several examples of 'experimental' complex networks it turns out to be 'scale-free', that is with a long power-law tail [5]. This implies that on an infinite scale-free graph nodes with diverging coordination are rather frequent, causing some subtleties in the application of the law of large numbers.

In this paper we concentrate our attention on homogeneous scale-free random trees subject only to the physically natural constraint of being embeddable in a finite-dimensional Euclidean 
space. This class of random trees has been studied already in [6, 7], although from a viewpoint different from ours: while in these works only some statistical averages relevant to characterize the geometry of these trees were analysed, we study here in depth the probability distributions of the basic observables which specify the intrinsic geometry. Our results apply therefore to any single 'generic' (infinite) scale-free random tree and not just to their statistical ensemble.

The outline of this paper is as follows. First of all we recall some definitions of graph theory and introduce the algorithm used to build homogeneous random trees, summarizing known results. The local connectivity dimension is then obtained from the scaling properties of the probability distributions for the volume and the surface of the spheres centred at the origin of the tree-constructing algorithm. Details of the scaled probabilities are studied and their asymptotic behaviour is determined. Then the probability distributions for the graph-averaged volume and the surface are considered; the average connectivity dimension is extracted from their scaling properties.

Our results for the growth properties of a random tree can be summarized in very concise heuristic formulae as follows: consider a sphere (intrinsically defined in terms of the chemical distance alone) of radius $r$ centred on some node; for large $r$ the volume $v$ of the sphere and the coordination fluctuation $\Delta z$ within the sphere are random variables simply related as

$$
v \simeq(\Delta z)^{2} r^{2}
$$

This relation applies to all random trees with bounded growth rate (see below) regardless of whether $\Delta z$ has a finite limit as $v \rightarrow \infty$ or not. In the former case, as happens for instance on trees with bounded coordination, one reads out immediately the connectivity dimension $d_{\mathrm{c}}=2$. In the latter case, one needs to estimate the asymptotic dependence of $\Delta z$ on $v$. For scale-free trees with tail exponent $2<\beta<3$ (see below for the proper definitions), one finds $\Delta z \sim v^{(3-\beta) /(\beta-1)}$, yielding the $\beta$-dependent connectivity dimension

$$
d_{\mathrm{c}}=\frac{\beta-1}{\beta-2} \text {. }
$$

Suppose instead that one is interested in the graph-averaged volume $\bar{v}$ obtained by averaging over all locations of the centre of the sphere (for a finite tree with large volume $V$ ). The random variable $\bar{v}$ is related to the coordination fluctuation $\overline{\Delta z}$ over the whole tree just as before, that is

$$
\bar{v} \simeq(\bar{\Delta} z)^{2} r^{2}
$$

Now the behaviour in $r$ is always quadratic, regardless of the way the coordinations are distributed. Of course, in the scale-free trees with $2<\beta<3$ one has $\overline{\Delta z} \sim V^{(3-\beta) /(\beta-1)}$, making it impossible to consider the standard thermodynamic limit $V \rightarrow \infty$. Nonetheless, we find that a well-defined limit exists for the 'renormalized' volume $\bar{v} V^{(\beta-3) /(\beta-1)}$. Therefore, in all cases we conclude for the average connectivity dimension

$$
\bar{d}_{\mathrm{c}}=2 \text {. }
$$

Finally, in the last section, the analysis of the probability distribution for the effective squared mass, defined through the Gaussian model on the tree, allows us to rigourously determine also the local spectral dimension $d_{\mathrm{s}}$. It fulfils the long-conjectured [8] relation $d_{\mathrm{s}}=2 d_{\mathrm{c}} /\left(d_{\mathrm{c}}+1\right)$ already verified in [7] through a general but completely different argument.

\section{Random trees}

\subsection{Generalities about graphs}

A graph $G$ is defined by a set of nodes (finite or countable), pairwise connected by a set of unordered links. If the set of nodes is finite, its cardinality will be denoted by $|G|$. The 
coordination number (or degree, or simply coordination) of a node $x$ is the number of its nearest neighbours and is denoted by $z_{x}$. The intrinsic properties of the graph are determined only by the connections between the nodes; the metric is given by the so-called chemical distance: the distance $d(x, y)$ is equal to the number of links of the shortest connected path between $x$ and $y$. This distance is used to define spheres; the 'volume' $v_{r}(x)$ is defined as the number of nodes of the radius $r$ sphere centred at node $x$ and the 'surface' $s_{r}(x)$ is the number of nodes of the corresponding spherical shell. Here we shall consider connected graphs in the limit of infinitely many nodes.

Given a function $F$ defined on the nodes, its average value $\bar{F}$ is defined as the infinite radius limit of the average over spheres; if such a limit exists, it does not depend on the centre of the spheres provided $F$ is bounded from below and the graph has a bounded growth rate [9], that is the surface vanishes with respect to the volume in the infinite size limit.

The properties of graphs can be described by various parameters; here we consider the connectivity dimension [10] and the spectral dimension [11]. The connectivity dimension describes how the volume of spheres scales with their radius $r$ for $r \rightarrow \infty$; we can define a local connectivity dimension $d_{\mathrm{c}}$ using the spheres centred on any given node $x$

$$
v_{r}(x) \sim r^{d_{\mathrm{c}}} .
$$

It is not difficult to show that $d_{\mathrm{c}}$ does not depend on $x$ when the graph has a bounded growth rate. We can define also an average dimension $\bar{d}_{\mathrm{c}}$ if the average volume is used (provided the limit defining it exists finite)

$$
\overline{v_{r}} \sim r^{\bar{d}_{\mathrm{c}}} .
$$

These two connectivity dimensions usually coincide, but on strongly inhomogeneous graphs they can be different [12].

The spectral dimension is related to long time properties of random walks on the graph, as well as to many other physical properties such as the infrared behaviour of the Gaussian model [13]. On a generic connected graph $G$ this model is defined by assigning a real-valued random variable $\phi_{x}$ to each node $x \in G$ with the Hamiltonian

$$
\mathcal{H}=\frac{1}{2} \mu_{0} \sum_{x} \phi_{x}^{2}+\frac{1}{2} \sum_{\langle x, y\rangle}\left(\phi_{x}-\phi_{y}\right)^{2}
$$

where $\langle x, y\rangle$ denotes nearest neighbour pairs of nodes and $\mu_{0}>0$ is a free parameter (the squared mass in the field-theoretic sense). The spectral dimension is determined by the infrared $\mu_{0} \rightarrow 0$ singularity of the diagonal element of the covariance

$$
\operatorname{Sing}\left|\left\langle\phi_{x}^{2}\right\rangle\right\rangle \sim \mu_{0}^{d_{\mathrm{s}} / 2-1}
$$

where $\langle\langle\cdot\rangle\rangle$ denotes standard Gibbs expectation values weighted with $\exp (-\mathcal{H})$. One can show that $d_{\mathrm{s}}$ does not depend on the choice of the node $x$ [13]. As for the connectivity dimension, it is possible to split the spectral dimension into a local one and an average one. The definition in equation (4) evidently corresponds to the local one; the average spectral dimension $\bar{d}_{\mathrm{s}}$ characterizes in the same way the singularity of the graph average of $\left\langle\left\langle\phi_{x}^{2}\right\rangle\right\rangle$. Again, the two dimensions usually coincide, but on strongly inhomogeneous graphs they can be different [12].

Suppose now that a certain statistical ensemble $\mathcal{G}$ of (infinite) graphs is given. As a consequence, connectivity and spectral dimensions become in principle random variables with probability distributions derived from the 'microscopic' one specifying $\mathcal{G}$. Consider the connectivity dimension: if the graphs of $\mathcal{G}$ are rooted, we can use the root, denoted by $o$, as the centre of the spheres whose growth defines $d_{\mathrm{c}}$; then the corresponding volumes $v_{r}(o)$ are 
random variables distributed with some probability $\operatorname{Pr}\left[v_{r}(o)=v\right]$. If this probability has a well-defined scaling behaviour for $r \rightarrow \infty$, that is

$$
\operatorname{Pr}\left[v_{r}(o)=v\right] \simeq \frac{1}{r^{d}} p\left(\frac{v}{r^{d}}\right)
$$

then the random variable $v_{r}(o) r^{-d}$ has a well-defined probability distribution in the limit $r \rightarrow \infty$ and we may write again

$$
v_{r}(o) \sim r^{d}
$$

as in equation (1), identifying $d$ with a non-fluctuating local connectivity dimension $d_{\mathrm{c}}$. In other words $d_{\mathrm{c}}$ is a property of a 'generic' specimen of the ensemble, that is a property of $\mathcal{G}$ itself. A similar argument applies to the average connectivity dimension $\bar{d}_{\mathrm{c}}$ and to the spectral dimensions using the random variable $\left\langle\left\langle\phi_{x}^{2}\right\rangle\right\rangle$ and its graph average.

An alternative approach is used in [6], where averages in the canonical or grand-canonical ensembles of certain random trees (i.e. graphs without loops) are studied rather than a single 'generic' sample tree. Two parameters are introduced to describe the intrinsic geometry of these trees, also called 'branched polymers': the Hausdorff dimensions $d_{H}$ and $d_{L}$. The former is related to how the average two-point distance on graphs with $V$ nodes scales with the size $V$ :

$$
\langle d(x, y)\rangle_{V} \sim V^{1 / d_{H}} .
$$

The latter (called local Hausdorff dimension in [6]) is related to the behaviour of the two-point correlation function $g_{V}^{(2)}(r)$ (proportional to the number of couples of nodes which are at distance $r$ ) for distances which are large, but much smaller than $V^{1 / d_{H}}$ :

$$
g_{V}^{(2)}(r) \stackrel{V \rightarrow \infty}{\sim} r^{d_{L}-1} \quad 1 \ll r \ll V^{1 / d_{H}} .
$$

It is claimed in [6] that $d_{H}$ and $d_{L}$ differ on a certain class of 'exotic' random trees characterized by unbounded local coordinations.

Actually, it is not a priori obvious if and how parameters such as $d_{H}$ and $d_{L}$ are connected to the connectivity dimensions $d_{\mathrm{c}}$ and $\bar{d}_{\mathrm{c}}$, although it is common lore to identify the Hausdorff dimension with the connectivity dimension when no distinction is made about local or average dimensions. One of the aims of the present work is just to fully answer this question in the case of homogeneous random trees, as we shall see below.

\subsection{Generalities about random trees}

Homogeneous random trees are built by the random independent extraction of every node's degree from a given distribution $f_{z}$. This process can be formulated as a Galton-Watson branching process [14]; in particular, since we are interested in trees with bounded growth rate, we must consider the critical Galton-Watson case. The given coordination distribution must be properly normalized

$$
\sum_{z} f_{z}=1
$$

and the average coordination must be equal to 2 because of the condition of bounded growth rate (see [9])

$$
\langle z\rangle=\sum_{z} z f_{z}=2
$$

Clearly these sums have a finite number of addends if the coordination is bounded, while they become a series if it is unbounded as in the scale-free case. The series, however, must be 
properly convergent in order for equations (7) and (8) to hold, so that $f_{z}$ must vanish faster than $z^{-2}$ for $z \rightarrow \infty$. Let us also introduce the probability generating function $g(\lambda)$,

$$
g(\lambda)=\sum_{z} f_{z} \lambda^{z-1}=f_{1}+f_{2} \lambda+f_{3} \lambda^{2}+\cdots
$$

which enjoys the properties

$$
g(1)=1 \quad g^{\prime}(1)=1
$$

easily derived from those of $f_{z}$.

A branching process effectively ends when a shell (that is a given generation of sibling branches) is made only by nodes with coordination 1 , so that the next shell is empty. The trees produced in this way are all finite because the surviving probability after $r$ generations vanishes for $r \rightarrow \infty$ in the critical Galton-Watson process [14]. Since we are interested in the thermodynamic limit we have two possibilities [9]. The first one is an explicit preconditioning on non-extinction: this means that modified branching probabilities are used to avoid finite trees, while keeping unaltered the properties of the infinite trees [15]. This is achieved if the root coordination is chosen with probability $f_{z}$ while on every successive shell the coordination of the first node is extracted with a modified probability distribution $\tilde{f}_{z}=(z-1) f_{z}$. The other possibility consists in calculating probabilities conditioned on the number of nodes $V$ of the resulting trees, and then take the limit $V \rightarrow \infty$. In this latter case the root coordination must be extracted with the refined probability

$$
\widehat{f}_{z}=N_{1} \frac{f_{z}}{z} \quad \frac{1}{N_{1}}=\sum_{z} \frac{f_{z}}{z}
$$

since the root has as many branches as its coordination while every other node has one branch less than its coordination (see section 3.1 of [9] for details).

\subsection{Trees with bounded coordination}

In [9] and [16] the geometrical and spectral properties of bounded random trees were determined using non-extinction preconditioning. The surface and volume probability, in the large radius limit, are shown to be functions only of the scaled variables $s / r$ and $v / r^{2}$, respectively. Moreover, after the discussion of the auto-averaging property, also average surface and volume are fully analysed. These results allow the rigourous determination of the connectivity dimension, both local and average: $d_{\mathrm{c}}=\bar{d}_{\mathrm{c}}=2$. The study of the Gaussian model on such trees leads also to the determination of the spectral dimension $d_{\mathrm{s}}=4 / 3$. Using a simple scaling hypothesis it is shown that the relation $d_{\mathrm{s}}=2 d_{\mathrm{c}} /\left(d_{\mathrm{c}}+1\right)$ should hold on a wider class of random trees.

An important universality property is also found: all the average values and probability distributions in the large radius limit depend on the $f_{z}$ distribution only through its second moment or, equivalently, $g^{\prime \prime}(1)$. This parameter quantifies the coordination fluctuations, since

$$
g^{\prime \prime}(1)=\sum_{z} f_{z}[z-\langle z\rangle]^{2}
$$

and characterizes all asymptotic behaviour. Altogether, these results can be summarized as

$$
v \simeq g^{\prime \prime}(1) r^{2}
$$

meaning that $v /\left[g^{\prime \prime}(1) r^{2}\right]$, as $r \rightarrow \infty$, is a random variable with a well-defined universal probability distribution.

The approach based on the grand-canonical ensemble of branched polymers yields the result $d_{H}=d_{L}=2$ when the coordination of each node is bounded. This corresponds to the 'generic phase' of random trees according to [6]. 


\subsection{Scale-free trees}

In this paper we turn to scale-free trees, that is trees whose coordination distribution has a long power-law tail:

$$
f_{z} \simeq A z^{-\beta} \quad \text { for } \quad z \rightarrow \infty
$$

where $\beta>2$. When the coordination is unbounded, the function $g(\lambda)$ may have singularities; in the scale-free case it becomes singular for $\lambda=1$. For any non-integer exponent ${ }^{1}$, its expansion contains a term proportional to $(1-\lambda)^{\beta-1}$, that is

$$
g(\lambda)=g_{a}(\lambda)+c_{*}(1-\lambda)^{\beta-1}
$$

where $g_{a}(\lambda)$ is analytic in $\lambda=1$

$$
g_{a}(\lambda)=1-(1-\lambda)+c_{2}(1-\lambda)^{2}+\cdots+c_{k}(1-\lambda)^{k}+\cdots
$$

and $c_{*}=A \Gamma(1-\beta)$. Therefore, if $2<\beta<3, g^{\prime \prime}(\lambda)$ diverges for $\lambda \rightarrow 1$; since we found $g^{\prime \prime}(1)$ to be an important parameter in the bounded coordination case, we expect this divergence to have many important consequences. As a first example it causes the divergence of $\left\langle v_{r}(o)\right\rangle$, the expected volume of the balls around the root of the branching process. This can be intuitively explained, using non-extinction preconditioning, because on every shell the coordination of one node is chosen with the probability distribution $\tilde{f}_{z}$, whose first moment is infinite. If auto-averaging holds, then also $\overline{v_{r}}$ would diverge on a single scale-free tree and the definition (2) of average connectivity dimension appears troublesome for scale-free trees. Thus, in order to deal with finite graph-averaged quantities, we will also use the finite volume approach, considering the volume $V$ as a regulator.

We may then find a heuristic argument based on equation (12) to determine the connectivity dimensions $d_{\mathrm{c}}$ and $\bar{d}_{\mathrm{c}}$ for scale-free random trees. First of all, if $\beta>3$ nothing changes because $g^{\prime \prime}(1)$ is still finite, so that $d_{\mathrm{c}}=\bar{d}_{\mathrm{c}}=2$. In contrast, if $2<\beta<3$, we have to face the divergence of $g^{\prime \prime}(1)$. But coordination fluctuations are finite for a finite number $N$ of nodes, and diverge with $N$ in a way fixed by the long tail of $f_{z}$, since all coordination extractions are independent. Let $z_{\max }(N)$ be the largest coordination extracted, estimated from

$$
\sum_{z=z_{\max }}^{\infty} f_{z} \sim z_{\max }^{1-\beta}=\frac{1}{N}
$$

then we may estimate fluctuations by

$$
\sum_{z=1}^{z_{\max }(N)}(z-\langle z\rangle)^{2} f_{z} \sim\left[z_{\max }(N)\right]^{3-\beta}=N^{v}
$$

where we defined the exponent $v$ as

$$
v=(3-\beta) /(\beta-1) \text {. }
$$

Thus for the local growth we have the consistency relation

$$
v_{r} \simeq v_{r}^{v} r^{2}
$$

which implies

$$
v_{r} \simeq r^{2 /(1-v)}=r^{(\beta-1) /(\beta-2)} \Rightarrow d_{\mathrm{c}}=\frac{\beta-1}{\beta-2} .
$$

When the graph-averaged $\overline{v_{r}}$ is considered for trees of $V$ nodes, the coordination fluctuation

1 For integer $\beta$ the singularity has a different, logarithmic, form; however, since the final results will be analytic in $\beta$, one is able to extend them also to integer values of $\beta$. 
over the whole graph should be used instead:

$$
\overline{v_{r}} \simeq r^{2} V^{(3-\beta) /(\beta-1)} .
$$

From this we read the scaling exponent 2 independently of $V$, but without the standard thermodynamic limit. Of course this is not a real proof and a more rigorous approach confirming these results is adopted in the following sections (see also appendix A); at any rate, these exponents are the same as those calculated for scale-free branched polymers with $2<\beta<3$ in the grand-canonical approach: $d_{H}=(\beta-1) /(\beta-2)$ [7] and $d_{L}=2$ [6].

\section{Growth statistics}

\subsection{Scaling probabilities for local surface and volume}

The probability $\operatorname{Pr}\left[s_{r}(o)=s\right]\left(\operatorname{Pr}\left[v_{r}(o)=v\right]\right)$ for the surface (volume) of the radius $r$ sphere around the root of an infinite tree can be found, recursively on $r$, using the modified probabilities given by explicit preconditioning (see [9], where a slightly different notation is used). The corresponding generating functions

$$
\begin{aligned}
G_{r}^{s}(\lambda) & =\sum_{s} \lambda^{s} \operatorname{Pr}\left[s_{r}(o)=s\right] \\
G_{r}^{v}(\lambda) & =\sum_{v} \lambda^{v} \operatorname{Pr}\left[v_{r}(o)=v\right]
\end{aligned}
$$

satisfy functional recursion rules easier to analyse, given by equations (15) and (16) in [9], that is

$$
\begin{aligned}
& G_{r+1}^{s}(\lambda)=\lambda g_{r+1}(\lambda) g_{r}^{\prime}(\lambda) \\
& \frac{G_{r+1}^{v}(\lambda, 1)}{G_{r}^{v}(\lambda, 1)}=\lambda g^{\prime}\left(h_{r-1}(\lambda)\right) \frac{h_{r+1}(\lambda)}{h_{r}(\lambda)}
\end{aligned}
$$

where

$$
\begin{array}{ll}
g_{r+1}(\lambda)=g\left(g_{r}(\lambda)\right) & g_{0}(\lambda)=\lambda \\
h_{r+1}(\lambda)=\lambda g\left(h_{r}(\lambda)\right) & h_{0}(\lambda)=\lambda .
\end{array}
$$

These recursions involve only $g(\lambda)$ and $g^{\prime}(\lambda)$, which are finite for $0 \leqslant \lambda \leqslant 1$ for every value of $\beta>2$, and therefore can be solved numerically with high accuracy for any $r$ (subject only to computational limits), thus providing a solution of our problem from a practical point of view. In figure 1 the $r=10$ volume probability corresponding to a tree with $\beta=2.5$ is plotted against the 'experimental' frequency distribution; this is obtained by sampling, over 1000 different graphs of $8 \times 10^{6}$ nodes each, the volume of 2000 balls centred on randomly chosen nodes. The agreement is good, and we notice the power-law tail with exponent that can be estimated as $\simeq-1.5$. In other cases (not shown) this is always equal to $1-\beta$.

Analytically, upon substitution of equations (13) and (14) for $g(\lambda)$, one can solve the recursion rules (15), (16) for the first terms of the expansions of $g_{r}(\lambda)$ and $h_{r}(\lambda)$ as $\lambda \rightarrow 1$ :

$$
\begin{aligned}
& g_{r}(\lambda) \simeq 1-(1-\lambda)+c_{2} r(1-\lambda)^{2}+\cdots+c_{*} r(1-\lambda)^{\beta-1} \\
& h_{r}(\lambda) \simeq 1+r(1-\lambda)+\frac{c_{2}}{3} r^{3}(1-\lambda)^{2}+\cdots+\frac{c_{*}}{\beta} r^{\beta}(1-\lambda)^{\beta-1}
\end{aligned}
$$

where only the leading order term in $r$ is shown for every power of $(1-\lambda)$. Similarly for $G_{r}^{s}(\lambda)$ and $G_{r}^{v}(\lambda)$ one finds

$$
\begin{aligned}
& G_{r}^{s}(\lambda) \simeq 1-2 c_{2} r(1-\lambda)+\cdots-(\beta-1) c_{*} r(1-\lambda)^{\beta-2} \\
& G_{r}^{v}(\lambda) \simeq 1-c_{2} r^{2}(1-\lambda)+\cdots-c_{*} r^{\beta-1}(1-\lambda)^{\beta-2} .
\end{aligned}
$$




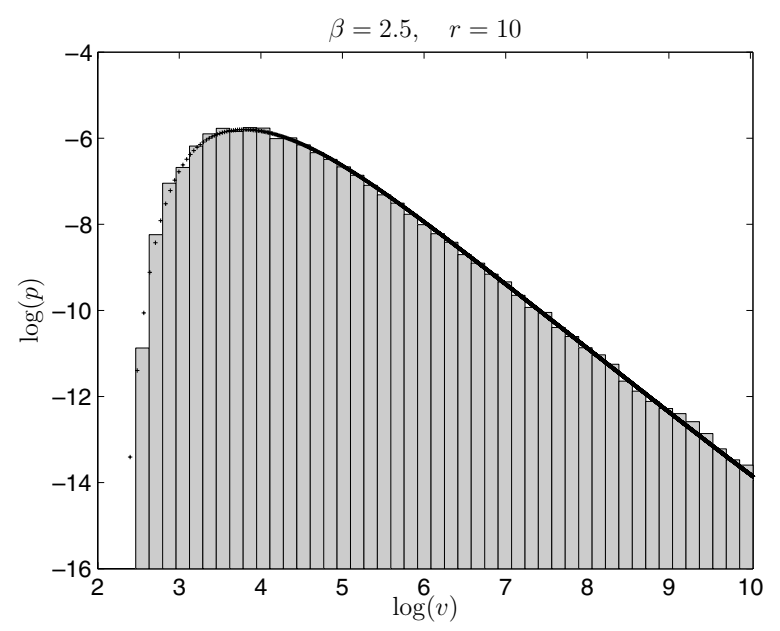

Figure 1. Numerically calculated volume distribution versus simulation histogram.

Through the rules of (inverse) discrete Laplace transform, the singular terms with noninteger powers in $1-\lambda$ determine power-law tails in the probability distributions at fixed $r$ :

$$
\operatorname{Pr}\left[s_{r}(o)=s\right] \stackrel{s \rightarrow \infty}{\simeq} A r s^{-(\beta-1)} \quad \operatorname{Pr}\left[v_{r}(o)=v\right] \stackrel{v \rightarrow \infty}{\simeq} \frac{A}{\beta-1} r^{\beta-1} v^{-(\beta-1)}
$$

in agreement with numerical data. The local connectivity dimension $d_{\mathrm{c}}$ is determined if we find scaling forms of these probabilities such that, as $s \rightarrow \infty$ or $v \rightarrow \infty$ and $r \rightarrow \infty$,

$$
\operatorname{Pr}\left[s_{r}(o)=s\right] \simeq \frac{1}{r^{d_{\mathrm{c}}-1}} \phi\left(\frac{s}{r^{d_{\mathrm{c}}-1}}\right) \quad \operatorname{Pr}\left[v_{r}(o)=v\right] \simeq \frac{1}{r^{d_{\mathrm{c}}}} \Phi\left(\frac{v}{r^{d_{\mathrm{c}}}}\right) .
$$

This means that $d_{\mathrm{c}}$ must be such that $G_{r}^{s}\left(\exp \left(-u / r^{d_{\mathrm{c}}-1}\right)\right.$ and $G_{r}^{v}\left(\exp \left(-\xi / r^{d_{\mathrm{c}}}\right)\right.$ have finite non-trivial limits for $r \rightarrow \infty$. The right scaling exponent is found by examining equation (18): if $\beta>3$ the leading order term in $(1-\lambda)$ is the linear one so that the finite functions of $u$ and $\xi$ are obtained with

$$
d_{\mathrm{c}}=2 \quad \text { for } \beta>3
$$

while if $2<\beta<3$ the most important term is the singular one so that the correct scaling is given by

$$
d_{\mathrm{c}}=\frac{\beta-1}{\beta-2} \quad \text { for } \quad 2<\beta<3 .
$$

In appendix $\mathrm{A}$ we present all explicit calculations about the surface and volume probability distributions, for the two cases $\beta>3$ and $\beta<3$, which confirm this simple scaling analysis.

\subsection{Fixed volume expectation values}

In this section we study the local growth properties of scale-free random trees of finite volume $V$. We shall reconstruct the dependence on $V$ of the surface and volume probability distributions from the expectation values of $s_{r}(o)$ and $v_{r}(o)$ and their powers. We are led to consider expectation values over finite-size trees, regarding their volume $V$ as a regulator, because the power-law tails with exponent $1-\beta$ found in the previous section for $\operatorname{Pr}\left[s_{r}(o)=s\right]$ and $\operatorname{Pr}\left[v_{r}(o)=v\right]$ imply the divergence with $V$ of high enough moments of $s_{r}(o)$ or $v_{r}(o)$. 
Let us observe that in this context the average is made over all possible rooted trees with $V$ nodes; this can also be seen as the graph average over all nodes of an unrooted tree, which is then further averaged over all possible realizations of the unrooted tree, that is

$$
\langle O\rangle_{V}=\langle\bar{O}\rangle_{V}
$$

for every observable $O$. In particular

$$
\left\langle s_{r}(o)^{n}\right\rangle_{V}=\left\langle\overline{s_{r}^{n}}\right\rangle_{V}
$$

with a similar relation for the volumes. No dependence on the root $o$ survives and we shall drop it from the expectation values.

Let us start by studying the probability $\widehat{P}(V)$ that any rooted tree $T$ with $V$ nodes is produced by the branching process without the non-extinction precondition

$$
\widehat{P}(V)=\operatorname{Pr}[|T|=V] .
$$

By the tree-producing algorithm, $\widehat{P}(V)$ satisfies the identity

$$
\widehat{P}(V)=\sum_{z} \widehat{f}_{z} \sum_{V_{1}, \ldots, V_{z}} \delta\left(V-1-\sum_{j=1}^{z} V_{j}\right) \prod_{j=1}^{z} P\left(V_{j}\right)
$$

where $P(V)=\operatorname{Pr}[|B|=V]$ is the same probability relative to a branch $B$, that is a tree whose root has an incoming link (and $z-1$ branches) and whose coordination is extracted with probability $f_{z}$; it satisfies a similar equation with $z-1$ instead of $z$ and $f_{z}$ instead of $\widehat{f}_{z}$ :

$$
P(V)=\sum_{z} f_{z} \sum_{V_{1}, \ldots, V_{z-1}} \delta\left(V-1-\sum_{j=1}^{z-1} V_{j}\right) \prod_{j=1}^{z-1} P\left(V_{j}\right) .
$$

The generating functions $\widehat{G}(\lambda)=\sum_{V} \widehat{P}(V) \lambda^{V}$ and $G(\lambda)=\sum_{V} P(V) \lambda^{V}$ satisfy

$$
G(\lambda)=\lambda g(G(\lambda)) \quad \widehat{G}(\lambda)=\lambda \widehat{g}(G(\lambda))
$$

where $g(\lambda)$ is the usual probability generating function defined in equation (9), while

$$
\widehat{g}(\lambda)=\sum_{z} \lambda^{z} \widehat{f}_{z}
$$

Normalization of $\widehat{f}_{z}$ implies $\widehat{g}(1)=1$ and from the definition it easily follows that $\widehat{g}(\lambda)$ derivatives are proportional to those of $g(\lambda)$; more precisely we have

$$
\widehat{g}^{(k)}(\lambda)=N_{1} g^{(k-1)}(\lambda) .
$$

Since we are interested in the $V \rightarrow \infty$ limit, which corresponds to $\lambda \rightarrow 1$, we can use expansions (13) and (14) to obtain the asymptotic behaviour of $G(\lambda)$ and $\widehat{G}(\lambda)$. For different values of $\beta$ we have different situations depending on which term, the singular one or the quadratic one, is of lower order.

For $\beta>3$ we have

$$
\begin{aligned}
& G(\lambda) \simeq 1-c_{2}^{-1 / 2}(1-\lambda)^{1 / 2} \\
& \widehat{G}(\lambda) \simeq 1-N_{1} c_{2}^{-1 / 2}(1-\lambda)^{1 / 2}
\end{aligned}
$$

so that, in the $V \rightarrow \infty$ limit, the probability $\widehat{P}(V)$ reads

$$
\widehat{P}(V) \simeq N_{1} \frac{1}{2 \sqrt{c_{2} \pi}} V^{-3 / 2} \text {. }
$$


For $2<\beta<3$, instead, the singular term produces

$$
\begin{aligned}
& G(\lambda) \simeq 1-c_{*}^{-1 /(\beta-1)}(1-\lambda)^{1 /(\beta-1)} \\
& \widehat{G}(\lambda) \simeq 1-N_{1} c_{*}^{-1 /(\beta-1)}(1-\lambda)^{1 /(\beta-1)} .
\end{aligned}
$$

Thus we have, for $V \rightarrow \infty$

$$
\widehat{P}(V) \simeq N_{1} \frac{-c_{*}^{-1 /(\beta-1)}}{\Gamma(-1 /(\beta-1))} V^{-\beta /(\beta-1)}
$$

Let us now turn to the calculation of the expected size of a shell of radius $r$ in trees with volume $V$. First of all, let us introduce the joint probability $\widehat{P}_{r}(s, V)$ that the size of the tree is $V$ and the $r$ th shell has $s$ nodes:

$$
\widehat{P}_{r}(s, V)=\operatorname{Pr}\left[s_{r}(o)=s,|T|=V\right] .
$$

It is related to the same probability relative to branches $P_{r}(s, V)$ as

$$
\widehat{P}_{r}(s, V)=\sum_{z} \widehat{f}_{z} \sum_{V_{1}, \ldots, V_{z}} \sum_{s_{1}, \ldots, s_{z}} \delta\left(V-1-\sum_{j=1}^{z} V_{j}\right) \delta\left(s-\sum_{j=1}^{z} s_{j}\right) \prod_{j=1}^{z} P_{r-1}\left(s_{j}, V_{j}\right)
$$

while $P_{r}(s, V)$ satisfies a similar recursion which is obtained from the previous one by replacing $z$ with $z-1$ and $\widehat{f}_{z}$ with $f_{z}$,

$P_{r}(s, V)=\sum_{z} f_{z} \sum_{V_{1}, \ldots, V_{z-1}} \sum_{s_{1}, \ldots, s_{z-1}} \delta\left(V-1-\sum_{j=1}^{z-1} V_{j}\right) \delta\left(s-\sum_{j=1}^{z-1} s_{j}\right) \prod_{j=1}^{z-1} P_{r-1}\left(s_{j}, V_{j}\right)$.

Clearly the expectation value of $s_{r}(o)$ on trees with $V$ nodes is now given by

$$
\left\langle s_{r}\right\rangle_{V}=\frac{\sum_{s} s \widehat{P}_{r}(s, V)}{\widehat{P}(V)}=\frac{\widehat{E}_{r}^{V}[s]}{\widehat{P}(V)}
$$

where $\widehat{E}_{r}^{V}[s]$ is defined as the weighted sum in the first numerator. Analogously we can define $E_{r}^{V}[s]$ as

$$
E_{r}^{V}[s]=\sum_{s} s P_{r}(s, V)
$$

and the two generating functions

$$
\widehat{E}_{r}^{\lambda}[s]=\sum_{V} \lambda^{V} \widehat{E}_{r}^{V}[s] \quad E_{r}^{\lambda}[s]=\sum_{V} \lambda^{V} E_{r}^{V}[s] .
$$

A recursion equation for the latter can be found using equation (23)

$$
\begin{aligned}
E_{r}^{\lambda}[s] & =\sum_{V} \lambda^{V} \sum_{s} s P_{r}(s, V) \\
& =\lambda \sum_{z} f_{z} \sum_{V_{1}, \ldots, V_{z-1}} \sum_{s_{1}, \ldots, s_{z-1}}\left(\sum_{k=1}^{z-1} s_{k}\right) \prod_{j=1}^{z-1} \lambda^{V_{j}} P_{r-1}\left(s_{j}, V_{j}\right) \\
& =\lambda \sum_{z} f_{z}(z-1) E_{r-1}^{\lambda}[s] G(\lambda)^{z-2} \\
& =\lambda g^{\prime}(G(\lambda)) E_{r-1}^{\lambda}[s]
\end{aligned}
$$

and one for $\widehat{E}_{r}^{\lambda}[s]$ is similarly obtained

$$
\widehat{E}_{r}^{\lambda}[s]=\lambda \widehat{g}^{\prime}(G(\lambda)) E_{r-1}^{\lambda}[s]=N_{1} \lambda g(G(\lambda)) E_{r-1}^{\lambda}[s] .
$$


Since $s_{0}(o)=1, E_{0}^{V}[s]$ is equal to $P(V)$, so that the initial condition reads

$$
E_{0}^{\lambda}[s]=G(\lambda) \text {. }
$$

Then, for $r \geqslant 0$, the solutions read

$$
\begin{aligned}
& E_{r}^{\lambda}[s]=G(\lambda)\left[\lambda g^{\prime}(G(\lambda))\right]^{r} \\
& \widehat{E}_{r}^{\lambda}[s]=N_{1} \lambda G(\lambda) g(G(\lambda))\left[\lambda g^{\prime}(G(\lambda))\right]^{r-1} .
\end{aligned}
$$

Now $\left\langle s_{r}\right\rangle_{V}$ can be easily found, but we have to consider the cases $\beta>3$ and $\beta<3$ separately.

For $\beta>3$ the $\lambda \rightarrow 1$ asymptotic expansion of $\widehat{E}_{r}^{\lambda}[s]$ reads

$$
\widehat{E}_{r}^{\lambda}[s] \simeq N_{1}\left[1-\left(2+2 c_{2}(r-1)\right) c_{2}^{-1 / 2}(1-\lambda)^{1 / 2}\right]
$$

which implies the large $V$ limit of $\left\langle s_{r}\right\rangle_{V}$

$$
\left\langle s_{r}\right\rangle_{V} \simeq 2+2 c_{2}(r-1) .
$$

For $2<\beta<3$ we have, instead,

$$
\widehat{E}_{r}^{\lambda}[s] \simeq N_{1}\left[1-(r-1)(\beta-1) c_{*}^{1 /(\beta-1)}(1-\lambda)^{(\beta-2) /(\beta-1)}\right]
$$

and

$$
\left\langle s_{r}\right\rangle_{V} \simeq k_{1}(\beta)(r-1) V^{v}
$$

where

$$
k_{1}(\beta)=(\beta-1) c_{*}^{2 /(\beta-1)} \frac{\Gamma(-1 /(\beta-1))}{\Gamma(-(\beta-2) /(\beta-1))} .
$$

In the first case the $V \rightarrow \infty$ limit is finite, while in the second one we find a divergence, as expected. To understand how equation (26) relates with the known $d_{\mathrm{c}}$, we now calculate higher moments of $s_{r}$. First of all, we generalize the notation $\widehat{P}_{r}(O, V), \widehat{E}_{r}^{V}[O]$ and $\widehat{E}_{r}^{\lambda}[O]$ to every observable $O$

$$
\begin{aligned}
& \widehat{P}_{r}(O, V)=\operatorname{Pr}\left[O_{r}(x)=O,|T|=V\right] \\
& \widehat{E}_{r}^{V}[O]=\sum_{O} O \widehat{P}_{r}(O, V) \\
& \widehat{E}_{r}^{\lambda}[O]=\sum_{V} \lambda^{V} \widehat{E}_{r}^{V}[O]
\end{aligned}
$$

together with their branch counterparts $P_{r}(O, V), E_{r}^{V}[O]$ and $E_{r}^{\lambda}[O]$. Now it is easy to show that the recurrence rule for $\widehat{E}_{r}^{\lambda}\left[s^{2}\right]$ reads

$$
\widehat{E}_{r}^{\lambda}\left[s^{2}\right]=\lambda \widehat{g}^{\prime}(G(\lambda)) E_{r-1}^{\lambda}\left[s^{2}\right]+\lambda \widehat{g}^{\prime \prime}(G(\lambda))\left(E_{r-1}^{\lambda}[s]\right)^{2} .
$$

Thus for $2<\beta<3$ the second moment is found to read

$$
\left\langle s_{r}^{2}\right\rangle_{V} \simeq k_{2}(\beta)(r-1) V^{\nu+1 /(\beta-1)}
$$

where $k_{2}(\beta)$ is some function of $\beta$ only.

For all higher moments $\widehat{E}_{r}^{\lambda}\left[s^{n}\right]$ we find, as a general rule, that the single $E_{r-1}^{\lambda}\left[s^{n}\right]$ on the right-hand side is multiplied by $\lambda \widehat{g}^{\prime}(G(\lambda))$ while the products of $k E_{r-1}^{\lambda}[\cdot]$ are multiplied by $\lambda \widehat{g}^{(k)}(G(\lambda))$. Moreover the equations for $E_{r}^{\lambda}[O]$ are the same as those for $\widehat{E}_{r}^{\lambda}[O]$ with $\widehat{g}(\cdot)$ substituted by $g(\cdot)$. Therefore we obtain for all higher moments, up to the leading terms in the $\lambda \rightarrow 1$ limit,

$$
\begin{aligned}
& \widehat{E}_{r}^{\lambda}\left[s^{n}\right] \simeq \lambda \widehat{g}^{\prime}(G(\lambda)) E_{r-1}^{\lambda}\left[s^{n}\right]+\cdots \\
& E_{r}^{\lambda}\left[s^{n}\right] \simeq \lambda g^{\prime}(G(\lambda)) E_{r-1}^{\lambda}\left[s^{n}\right]+\lambda g^{(n)}(G(\lambda)) E_{r-1}^{\lambda}[s]^{n}+\cdots
\end{aligned}
$$




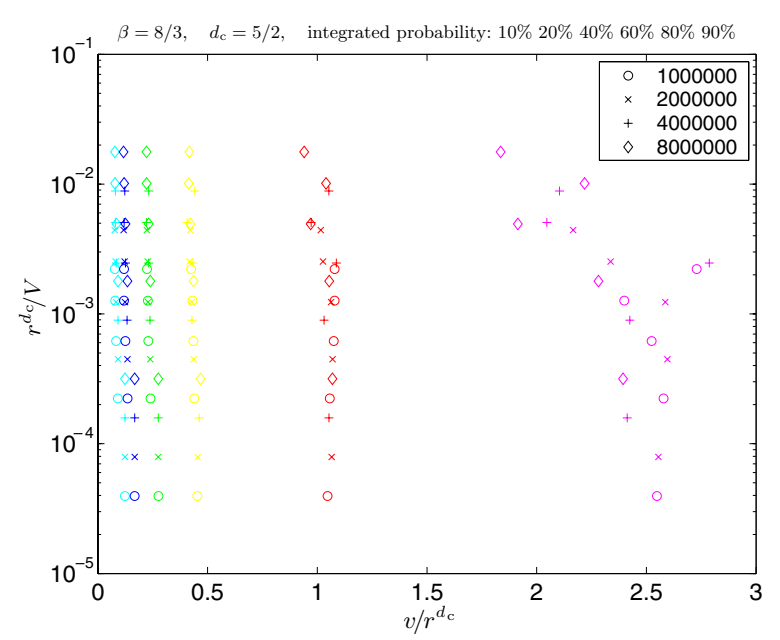

Figure 2. The symbols correspond to all possible combinations of $r=10,20,30,40,50$ (using the same symbol) and $V=1,2,4,8 \times 10^{6}$ (using different symbols as explained in the legend).

(This figure is in colour only in the electronic version)

This entails the final result

$$
\left\langle s_{r}^{n}\right\rangle_{V} \simeq k_{n}(\beta)(r-1) V^{\nu+(n-1) /(\beta-1)}
$$

which is compatible with a scaling form for the $s_{r}$ probability just given by a finite-size scaling of the one found in section 3.1 , that is

$$
\widehat{P}_{r}(s \mid V)=\frac{\widehat{P}_{r}(s, V)}{\widehat{P}(V)} \simeq \frac{1}{r^{d_{\mathrm{c}}-1}} q\left(\frac{s}{r^{d_{\mathrm{c}}-1}}, \frac{r^{d_{\mathrm{c}}}}{V}\right)
$$

where $d_{\mathrm{c}}$ is the already known local connectivity dimension

$$
d_{\mathrm{c}}=\frac{\beta-1}{\beta-2}
$$

and the function $q(x, y)$ is such that

$$
\int x^{n} q(x, y) \mathrm{d} x \simeq y^{(\beta-2-n) /(\beta-1)} .
$$

Similarly it is possible to calculate the finite volume moments of the volume $v_{r}(o)$ which read

$$
\left\langle v_{r}^{n}\right\rangle_{V} \sim r^{n+1} V^{(n+2-\beta) /(\beta-1)} .
$$

The proper scaling compatible with this result is

$$
\widehat{P}_{r}(v \mid V) \simeq \frac{1}{r^{d_{\mathrm{c}}}} p\left(\frac{v}{r^{d_{\mathrm{c}}}}, \frac{r^{d_{\mathrm{c}}}}{V}\right)
$$

if the moments of the scaling function $p(x, y)$ behave as

$$
\int x^{n} p(x, y) \mathrm{d} x \simeq y^{(\beta-2-n) /(\beta-1)} .
$$

Scaling (29) agrees with numerical simulations as shown in figure 2. For several different values of $r$ and $V$, we built 720 trees and calculated the volume $v$ of radius $r$ balls around 1000 
(for each tree) randomly chosen nodes. Thus different 'experimental' probability distributions are found; we considered the integrated probability $Q_{r}(v \mid V)=\sum_{v^{\prime} \leqslant v} \widehat{P}_{r}(v \mid V)$ and plotted the values of $v$ corresponding to $Q_{r}(v \mid V)=0.1,0.2,0.4,0.6,0.8,0.9$ (after the proper rescaling by $\left.r^{d_{\mathrm{c}}}\right)$ versus $r^{d_{\mathrm{c}}} / V$. For every one of the six values of $Q_{r}(v \mid V)$, the points corresponding to different values of $r$ and $V$ fall roughly on a curve, apart from statistical errors (fluctuations are especially large for $Q_{r}(v \mid V)=0.9$, due to the long power-law tail of $\widehat{P}_{r}(v \mid V)$ ), so that the form (29) of the probability scaling is supported.

\subsection{Probability of the average surface}

In the bounded coordination case we were able to prove the auto-averaging property for every local observable: the graph average $\bar{O}$ is non-fluctuating on infinite trees and coincides with the expectation value $\langle O\rangle$ in the branching process around the origin [9, 16]. This implies immediately that local and average connectivity dimensions coincide.

In the scale-free case the situation is more involved because many relevant expectation values do not even exist on infinite trees, so that we cannot prove the auto-averaging property in the same way. Thus, to determine the average connectivity dimension $\bar{d}_{\mathrm{c}}$ we will try to directly investigate the probability distribution of the average surface $\overline{s_{r}}$ in the large $r$ limit. In particular, we look for a scaling form of the type

$$
\operatorname{Pr}\left[\overline{s_{r}}=s\right] \simeq \frac{1}{r^{\bar{d}_{\mathrm{c}}-1}} f\left(\frac{s}{r^{\bar{d}_{\mathrm{c}}-1}}\right) .
$$

A very close approach is possible also for the average volume $\overline{v_{r}}$, but it is more involved and will not be reported here.

The results of the previous section, together with equation (20), allow us to calculate only the first moments of such a probability distribution. We now reconstruct it (at least its scaling form) through the calculation of higher moments.

This can be done by introducing $S_{r}$, defined as the sum of $s_{r}(x)$ over all nodes $x$ of a tree:

$$
S_{r}=\sum_{x \in T} s_{r}(x) .
$$

Then on a finite-sized tree, the average surface $\overline{s_{r}}$ is given by

$$
\overline{s_{r}}=\frac{S_{r}}{V}
$$

and its (finite-volume) moments are simply obtained,

$$
\left\langle\left(\overline{s_{r}}\right)^{n}\right\rangle_{V}=\frac{\left\langle S_{r}^{n}\right\rangle_{V}}{V^{n}}
$$

The recursive nature of the trees allows us again to find the composition rule for $S_{r}$ in terms of the values of $S_{r, j}$ and the local surface $s_{k, j}$ (with $k<r$ ) relative to the $j$ th branch around the root $o$. It reads

$$
S_{r}=\sum_{j}\left[S_{r, j}+2 s_{r-1, j}+\sum_{k=0}^{r-2} \sum_{j^{\prime} \neq j} s_{k, j} s_{r-k-2, j^{\prime}}\right] .
$$

The recursion rule for the joint probability of $S_{r}, s_{1}, \ldots, s_{r-1}$ and $V$ easily follows from (30) and the recursion rules for $s_{r}$ and $V$. With calculations similar to those of the previous section one obtains

$$
\widehat{E}_{r}^{\lambda}[S]=\lambda \widehat{g}^{\prime}(G(\lambda)) E_{r}^{\lambda}[S]+2 \lambda \widehat{g}^{\prime}(G(\lambda)) E_{r-1}^{\lambda}[s]+\lambda \widehat{g}^{\prime \prime}(G(\lambda)) \sum_{k=0}^{r-2} E_{k}^{\lambda}[s] E_{r-k-2}^{\lambda}[s]
$$


and, as usual, the equation for $E_{r}^{\lambda}[S]$ which is equal to this one with all $\widehat{g}(\cdot)$ substituted by $g(\cdot)$. The expectation value

$$
\left\langle\overline{s_{r}}\right\rangle_{V}=\frac{\widehat{E}_{r}^{\lambda}[S]}{V \widehat{P}(V)}
$$

is then obtained by straightforward calculations in the $V \rightarrow \infty$ limit, and the result is equal to the large $V$ limit of $\left\langle s_{r}\right\rangle_{V}$, as expected (recall equation (20)).

The differences of course emerge when higher moments are considered, since $\overline{s_{r}^{n}} \neq\left(\overline{s_{r}}\right)^{n}$ for $n>1 .\left\langle\left(\overline{s_{r}}\right)^{2}\right\rangle_{V}$ can be obtained using the equations (only the leading terms are displayed) $\widehat{E}_{r}^{\lambda}\left[S^{2}\right] \simeq \lambda \widehat{g}^{\prime}(G(\lambda)) E_{r}^{\lambda}\left[S^{2}\right]+\cdots$

$$
\begin{aligned}
& E_{r}^{\lambda}\left[S^{2}\right] \simeq \lambda g^{\prime}(G(\lambda)) E_{r}^{\lambda}\left[S^{2}\right]+\lambda g^{\prime \prime}(G(\lambda)) E_{r}^{\lambda}[S]^{2} \\
& +\lambda g^{(4)}(G(\lambda)) \sum_{k=0}^{r-2} \sum_{k^{\prime}=0}^{r-2} E_{k}^{\lambda}[s] E_{k^{\prime}}^{\lambda}[s] E_{r-k-2}^{\lambda}[s] E_{r-k^{\prime}-2}^{\lambda}[s]+\cdots
\end{aligned}
$$

and turns out to read

$$
\left\langle\left(\overline{s_{r}}\right)^{2}\right\rangle_{V} \simeq \begin{cases}\left(2+2 c_{2}(r-1)\right)^{2} & \text { for } \beta>3 \\ \bar{k}_{2}(\beta)(r-1)^{2} V^{2 v} & \text { for } 2<\beta<3\end{cases}
$$

where $\bar{k}_{2}(\beta)$ does not depend on $r$ or $V$.

Then all the following moments can be calculated through the equations

$$
\begin{aligned}
& \widehat{E}_{r}^{\lambda}\left[S^{n}\right] \simeq \lambda \widehat{g}^{\prime}(G(\lambda)) E_{r}^{\lambda}\left[S^{n}\right]+\cdots \\
& E_{r}^{\lambda}\left[S^{n}\right] \simeq \lambda g^{\prime}(G(\lambda)) E_{r}^{\lambda}\left[S^{n}\right]+\lambda g^{\prime \prime}(G(\lambda)) \frac{1}{2} \sum_{j} \frac{n !}{j !(n-j) !} E_{r}^{\lambda}\left[S^{j}\right] E_{r}^{\lambda}\left[S_{r}^{n-j}\right] \\
& \quad+\lambda g^{\prime \prime \prime}(G(\lambda)) \frac{1}{3 !} \sum_{j, j^{\prime}} \frac{n !}{j ! j^{\prime} !\left(n-j-j^{\prime}\right) !} E_{r}^{\lambda}\left[S^{j}\right] E_{r}^{\lambda}\left[S^{j^{\prime}}\right] E_{r}^{\lambda}\left[S^{n-j-j^{\prime}}\right] \\
& \quad+\cdots+\lambda g^{(n)}(G(\lambda)) E_{r}^{\lambda}[S]^{n} \\
& +\lambda g^{(2 n)}(G(\lambda)) \sum_{k_{1}, \ldots, k_{n}} E_{k_{1}}^{\lambda}[s] E_{r-k_{1}-2}^{\lambda}[s] \cdots E_{k_{n}}^{\lambda}[s] E_{r-k_{n}-2}^{\lambda}[s]+\cdots
\end{aligned}
$$

From the leading singularity in $1-\lambda$, we read their asymptotic behaviour for large $V$ :

$$
\left\langle\left(\overline{s_{r}}\right)^{n}\right\rangle_{V} \simeq \begin{cases}\left(2+2 c_{2}(r-1)\right)^{n} & \text { for } \beta>3 \\ \bar{k}_{n}(\beta)(r-1)^{n} V^{n v} & \text { for } 2<\beta<3 .\end{cases}
$$

For $\beta>3$, since all moments are powers of the same finite quantity, the probability distribution for $\overline{s_{r}}$ must be a delta function in the $V \rightarrow \infty$ limit

$$
\operatorname{Pr}\left[\overline{s_{r}}=s\right]=\delta\left(s-\left(2+2 c_{2}(r-1)\right)=\delta\left(s-\left\langle s_{r}\right\rangle\right) .\right.
$$

Thus in the thermodynamic limit the average does not fluctuate and coincides with the expectation value for the surface around the root of the tree and the auto-averaging property is verified for the observable $s_{r}$. In particular we can extract the average connectivity dimension

$$
\bar{d}_{\mathrm{c}}=d_{\mathrm{c}}=2 \text {. }
$$

Similarly the auto-averaging property can be verified for the volume; we expect it to hold in general for every observable with a finite expectation value.

When $2<\beta<3$ instead, from equation (32) we can read the following scaling form for the average surface probability:

$$
\widehat{P}_{r}(\bar{s} \mid V) \simeq \frac{1}{r V^{\nu}} \bar{q}\left(\frac{\bar{s}}{r V^{\nu}}\right)
$$


This shows that for every $V$ there exists a scaling form of the probability distribution of $\bar{s}$ for all scale-free trees of size $V$, but it does not have a non-trivial standard thermodynamic limit. Although $\overline{s_{r}}$ diverges on infinite trees and the standard definition of average connectivity dimension cannot be applied, we see that a $V$-independent probability exists for the 'renormalized' average surface $\bar{s} V^{-v}$ and that this scales according to the average connectivity dimension $\bar{d}_{\mathrm{c}}=2$.

For finite trees a comparison can be made with the definition of $d_{L}$ (equation (6)): since the average surface differs from the two-point function only through a normalization factor

$$
g_{V}^{(2)}(r) \propto\left\langle s_{r}\right\rangle_{V} \propto r
$$

our calculation not only confirms the value $d_{L}=2$; it also shows that this is not a property of the first moment only, but it holds 'in probability', that is for every 'generic' scale-free random tree.

\section{Spectral dimension}

In order to find the (local) spectral dimension of scale-free trees, we may repeat the approach of [16] substituting fixed radius averages with fixed volume averages. The quantity $\left\langle\left\langle\phi_{x}^{2}\right\rangle\right\rangle$ is given by a normalized Gaussian integral over all variables $\phi_{y}, y \in G$; if we perform all integrations except the one over $\phi_{x}$, we are left with a last integral which is also Gaussian and normalized, thanks to the self-reproducing property of Gaussian integrals. Therefore we can define the effective squared mass $\mu(x)$ from the width of this last integral:

$$
\left\langle\left\langle\phi_{x}^{2}\right\rangle\right\rangle=\sqrt{\frac{\mu(x)}{2 \pi}} \int \mathrm{d} \phi_{x} \phi_{x}^{2} \mathrm{e}^{-\mu(x) \phi_{x}^{2} / 2}=\frac{1}{2 \mu(x)} .
$$

On a tree produced by a branching process the rules of Gaussian integration allow us to express the effective squared mass of the root as a function of those of the branches [16]:

$$
\mu(x)=\mu_{0}+\sum_{\substack{y \text { branches } \\ \text { of } x}} \frac{\mu(y)}{1+\mu(y)} .
$$

Similarly the effective squared mass of a branch can be expressed as a function of those of its sub-branches, in a recursive way. Since the recursion rule for $\mu(x)$ is highly nontrivial we are not able to find directly its probability distribution or its moments; instead we expand $\mu(x)$ in powers of $\mu_{0}$ and consider the coefficients $V_{n}$

$$
\mu(x)=\sum_{n=1}^{\infty}(-1)^{n+1} V_{n}(x) \mu_{0}^{n} .
$$

Substituting this expansion in equation (34), recursion rules for the coefficients are obtained:

$$
\begin{aligned}
& V_{1}(x)=1+\sum_{\substack{y \text { branches } \\
\text { of } x}} V_{1}(y) \\
& V_{2}(x)=\sum_{\substack{y \text { branches } \\
\text { of } x}}\left[V_{2}(y)+V_{1}(y)^{2}\right]
\end{aligned}
$$

and, in general

$$
V_{n}(x)=\delta_{n, 1}+\sum_{\substack{y \text { branches } \\ \text { of } x}}\left[V_{n}(y)+\mathcal{F}_{n}\left(V_{1}(y), V_{2}(y), \ldots, V_{n-1}(y)\right)\right]
$$


where $\mathcal{F}_{n}$ is explicitly given in appendix B. The important feature which allows the calculation of $V_{n}$ moments is that the expression of $V_{n}(x)$ is linear in the $V_{n}(y)$.

Clearly $V_{1}$ is just the volume $V$ so that

$$
\left\langle V_{1}\right\rangle_{V}=V \quad \text { and } \quad\left\langle V_{1}^{n}\right\rangle_{V}=V^{n} .
$$

Now we need the averages $\left\langle V_{n}\right\rangle_{V}$ for $n \geqslant 2$. We use the same notation as in the previous sections $\left(\widehat{P}(\cdot), \widehat{E}^{V}[\cdot], \widehat{E}^{\lambda}[\cdot], P(\cdot), \ldots\right)$ with the only difference that now observables do not depend on $r$.

Let us start with the expectation value of $V_{2}$; equations for $\widehat{E}^{\lambda}\left[V_{2}\right]$ and $E^{\lambda}\left[V_{2}\right]$ can be easily obtained in the same way as before,

$$
\begin{aligned}
\widehat{E}^{\lambda}\left[V_{2}\right] & =\lambda \widehat{g}^{\prime}(G(\lambda))\left[E^{\lambda}\left[V_{2}\right]+E^{\lambda}\left[V_{1}^{2}\right]\right] \\
E^{\lambda}\left[V_{2}\right] & =\lambda g^{\prime}(G(\lambda))\left[E^{\lambda}\left[V_{2}\right]+E^{\lambda}\left[V_{1}^{2}\right]\right] \\
& =\left[1-\lambda g^{\prime}(G(\lambda))\right]^{-1} \lambda g^{\prime}(G(\lambda)) E^{\lambda}\left[V_{1}^{2}\right] .
\end{aligned}
$$

This is valid for any $f_{z}$ distribution, but now the $2<\beta<3$ and $\beta>3$ cases must be examined separately.

When $2<\beta<3$ we can read the asymptotic behaviour of $\left\langle V_{2}\right\rangle$ from the leading $1-\lambda$ singularity in equation (35):

$$
\left\langle V_{2}\right\rangle_{V}=\frac{\widehat{E}^{V}\left[V_{2}\right]}{\widehat{P}(V)} \sim V^{(3 \beta-4) /(\beta-1)} \quad V \rightarrow \infty .
$$

In a similar way it can be proved that (see appendix B for details)

$$
\left\langle V_{n}\right\rangle_{V}=\frac{\widehat{E}^{V}\left[V_{n}\right]}{\widehat{P}(V)} \sim V^{1+(n-1)(2 \beta-3) /(\beta-1)} \quad V \rightarrow \infty .
$$

We can then write

$$
\langle\mu\rangle_{V} \simeq \mu_{0} V F_{1}\left(\mu_{0} V^{(2 \beta-3) /(\beta-1)}\right) .
$$

The existence of the thermodynamic limit [13] requires that $F_{1}(t) \sim t^{-(\beta-1) /(2 \beta-3)}$ for $t \rightarrow \infty$ so that the powers of $V$ cancel out and a finite limit is obtained. After the $V \rightarrow \infty$ limit, for $\mu_{0} \rightarrow 0$ we have (see section 5 of [16] for a discussion on the order of the limits)

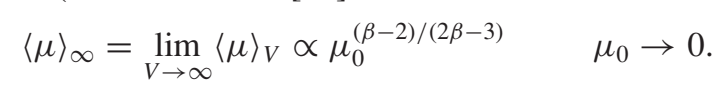

Equation (B3) in appendix B can be used to show that the higher moments of $\mu$ follow similar scaling laws:

$$
\left\langle\mu^{n}\right\rangle_{V} \simeq \mu_{0}^{n} V^{n} F_{n}\left(\mu_{0} V^{(2 \beta-3) /(\beta-1)}\right)
$$

so that

$$
\left\langle\mu^{n}\right\rangle_{\infty} \sim \mu_{0}^{n(\beta-2) /(2 \beta-3)} \quad \mu_{0} \rightarrow 0
$$

Since all moments are proportional to powers of the same quantity, we can say that there exists a limit probability distribution for the scaled variable $\mu \mu_{0}^{-(\beta-2) /(2 \beta-3)}$ so that the local spectral dimension reads

$$
d_{\mathrm{s}}=2 \frac{\beta-1}{2 \beta-3} \quad \text { for } \quad 2<\beta<3
$$

When $\beta>3$ equation (35) allows us to write

$$
\left\langle V_{2}\right\rangle_{V}=\frac{\widehat{E}^{V}\left[V_{2}\right]}{\widehat{P}(V)} \sim V^{5 / 2} \quad V \rightarrow \infty .
$$


All other moments are calculated in appendix B and, following the same steps as before, we can write

$$
\left\langle\mu^{n}\right\rangle_{\infty} \sim \mu_{0}^{n / 3} \quad \mu_{0} \rightarrow 0
$$

so that

$$
d_{\mathrm{s}}=\frac{4}{3} \quad \text { for } \quad \beta>3
$$

as in the bounded coordination case [16].

Finally, we note that in both cases the relation

$$
d_{\mathrm{s}}=2 \frac{d_{\mathrm{c}}}{d_{\mathrm{c}}+1}
$$

between the connectivity and the spectral dimension is fulfilled.

\section{Appendix A. Details on probability distributions}

In order to find the probability distributions for surface and volume we can follow the same steps of [9]. If the proper scaling forms of the surface and volume generating functions are substituted in their recursion rules, differential equations are obtained by consistency requirements. The solutions are the Laplace transform of the scaled surface and volume probability distributions. For $\beta>3$ both Laplace transforms can be calculated, while for $2<\beta<3$ only the surface probability Laplace transform and the asymptotic behaviour of the volume can be obtained.

\section{A.1. $\beta>3$}

When $\beta>3, d_{\mathrm{c}}$ is equal to 2 and we can write $g_{r}(\lambda)$ as

$$
g_{r}\left(\mathrm{e}^{-u / r}\right)=1-\frac{a(u)}{r}+\cdots+\frac{a_{*}(u)}{r^{\beta-2}}+\cdots
$$

with the condition

$$
a(0)=0 \quad a^{\prime}(0)=1
$$

which follows from $g_{r}(1)=g_{r}^{\prime}(1)=1$ and

$$
a_{*}(u) \simeq c_{*} u^{\beta-1} \quad \text { for } \quad u \rightarrow 0
$$

derived from equation (17). This expansion implies those of $G_{r}^{s}(\lambda)$ and $\operatorname{Pr}\left[s_{r}(o)=s\right]$ :

$$
\begin{aligned}
& G_{r}^{s}\left(\mathrm{e}^{-u / r}\right)=a^{\prime}(u)+\cdots-\frac{a_{*}^{\prime}(u)}{r^{\beta-3}}+\cdots \\
& \operatorname{Pr}\left[s_{r}(o)=s\right]=\frac{1}{r} \phi\left(\frac{s}{r}\right)+\cdots+\frac{1}{r^{\beta-2}} \phi_{*}\left(\frac{s}{r}\right)+\cdots
\end{aligned}
$$

where $\phi(x)$ and $\phi_{*}(x)$ are the inverse Laplace transforms of $a^{\prime}(u)$ and $a_{*}^{\prime}(u)$, respectively. The analytic term $\phi(x)$ is the same as in the bounded case; the singular term $\phi_{*}(x)$, even if it is suppressed by powers of $r$, is essential in order to reproduce the power-law tail. The functions $a(u)$ and $a_{*}(u)$ are determined by inserting equation (A1) in the recurrence (15), and equating the coefficients of the corresponding powers of $r$. Thus two coupled differential equations are obtained (see [9] for details on the method in the case of bounded trees, when only $a(u)$ is required):

$$
\begin{aligned}
& u a^{\prime}(u)=a(u)-c_{2} a(u)^{2} \\
& u a_{*}^{\prime}(u)=(\beta-2) a_{*}(u)-2 c_{2} a(u) a_{*}(u)+c_{*} a(u)^{\beta-1}
\end{aligned}
$$


The solution of the former, obtained with the use of the initial condition (A2), reads

$$
a(u)=\frac{u}{1+c_{2} u}
$$

while the general solution of the latter reads

$$
a_{*}(u)=\frac{c_{*}}{c_{2}(\beta-4)} u^{\beta-2}\left[\frac{k}{\left(1+c_{2} u\right)^{2}}-\frac{1}{\left(1+c_{2} u\right)^{\beta-2}}\right] .
$$

The value of $k$ is found by comparison with condition (A3) and turns out to be $k=1$. The inverse Laplace transforms $\phi(x)$ of $a^{\prime}(u)$ is

$$
\phi(x)=\frac{x}{c_{2}^{2}} \mathrm{e}^{-x / c_{2}}
$$

while only the asymptotic $x \rightarrow \infty$ behaviour of $\phi_{*}(x)$ can be explicitly calculated:

$$
\phi_{*}(x) \simeq A x^{-(\beta-1)} .
$$

Therefore, even if the contribution of $\phi_{*}(x)$ in equation (A4) is suppressed by powers of $r$, it is the most important one for large $x$ because of its power-law tail compared with the exponential one of $\phi(x)$. Moreover, if $\beta>4$ there are other lower order corrections before the singular one; however, since their contribution in the Laplace transform $G_{r}^{s}(\exp (u / r))$ is analytic for $u \rightarrow 0$, they are exponentially vanishing for large $s / r$. The large $s$ tail is therefore due to the singular term.

Similar calculations can also be done for the volume probability. The first step consists in writing the expansion of $h_{r}(\lambda)$ with the proper scaling:

$$
h_{r}\left(\mathrm{e}^{-\xi / r^{2}}\right)=1-\frac{b(\xi)}{r}+\cdots+\frac{b_{*}(\xi)}{r^{\beta-2}}+\cdots
$$

with the condition

$$
b(0)=0 \quad b^{\prime}(0)=1
$$

which comes from $h_{r}(1)=1$ and $h_{r}^{\prime}(1)=r$ and

$$
b_{*}(\xi) \simeq \frac{c_{*}}{\beta} u^{\beta-1}
$$

derived from equation (17). Inserting equation (A5) in the recurrence for $h_{r}(\lambda)$ (equation (16)) and equating the coefficients of corresponding powers of $r$, we obtain

$$
\begin{aligned}
& 2 \xi b^{\prime}(\xi)=b(\xi)-c_{2} b(\xi)^{2}+\xi \\
& 2 \xi b_{*}^{\prime}(\xi)=(\beta-2) b_{*}(\xi)-2 c_{2} b(\xi) b_{*}(\xi)+c_{*} b(\xi)^{\beta-1} .
\end{aligned}
$$

Their solutions, imposing conditions (A6) and (A7), can be found to read

$$
\begin{aligned}
& b(\xi)=\sqrt{\frac{\xi}{c_{2}}} \tanh \sqrt{c_{2} \xi} \\
& b_{*}(\xi)=\frac{c_{*}}{2 \xi}\left(\sqrt{\frac{\xi}{c_{2}}} \tanh \sqrt{c_{2} \xi}\right)^{\beta}\left[1-\frac{\beta-2}{\beta} \frac{{ }_{2} F_{1}\left(1, \beta / 2 ; 1+\beta / 2 ;\left(\tanh \sqrt{c_{2} \xi}\right)^{2}\right)}{\left(\cosh \sqrt{c_{2} \xi}\right)^{2}}\right]
\end{aligned}
$$

where ${ }_{2} F_{1}(a, b ; c ; z)$ is the hyper-geometric function; in our case it can also be written as

$$
{ }_{2} F_{1}(1, b ; 1+b ; z)=\sum_{n=0}^{\infty} \frac{b}{b+n} z^{n} .
$$


The volume probability $\operatorname{Pr}\left[v_{r}(o)=v\right]$ and its generating function $G_{r}^{v}(\lambda)$ now can be written as

$$
\begin{aligned}
& G_{r}^{v}\left(\mathrm{e}^{-\xi / r^{2}}\right)=l(\xi)\left(1+\cdots-\frac{l_{*}(\xi)}{r^{\beta-3}}+\cdots\right) \\
& \operatorname{Pr}\left[v_{r}(o)=v\right]=\frac{1}{r^{2}} \Phi\left(\frac{v}{r^{2}}\right)+\cdots+\frac{1}{r^{\beta-1}} \Phi_{*}\left(\frac{v}{r^{2}}\right)+\cdots
\end{aligned}
$$

where $\Phi(x)$ and $\Phi_{*}(x)$ are the inverse Laplace transforms of $l(\xi)$ and $l(\xi) l_{*}(\xi)$, respectively. Probability normalization requires $l(0)=1$ and $l_{*}(0)=0$; the functions $l(\xi)$ and $l_{*}(\xi)$ are related to $b(\xi)$ and $b_{*}(\xi)$ by the differential equations:

$$
\begin{aligned}
& \xi l^{\prime}(\xi)=-c_{2} b(\xi) l(u) \\
& 2 \xi l_{*}^{\prime}(\xi)=(\beta-3) l_{*}(\xi)-2 c_{2} b_{*}(\xi)+c_{*}(\beta-1) b(\xi)^{\beta-2} .
\end{aligned}
$$

The solution of the former is found in [9] and reads

$$
l(\xi)=\frac{1}{\left(\cosh \sqrt{c_{2} \xi}\right)^{2}}
$$

while for the latter we obtain

$$
\begin{gathered}
l_{*}(\xi)=\frac{c_{*}}{c_{2}}\left(\frac{\xi}{c_{2}}\right)^{(\beta-3) / 2}\left[t^{\beta-1}{ }_{2} F_{1}\left(1, \frac{\beta-1}{2} ; \frac{\beta+1}{2} ; t^{2}\right)+t^{\beta+1} \frac{\beta-1}{\beta+1}{ }_{2} F_{1}\left(1, \frac{\beta+1}{2} ; \frac{\beta+3}{2} ; t^{2}\right)\right. \\
\left.-t^{\beta+1} \frac{\beta-2}{\beta}{ }_{2} F_{1}\left(1, \frac{\beta}{2} ; 1+\frac{\beta}{2} ; t^{2}\right)\right]
\end{gathered}
$$

where $t=\tanh \sqrt{c_{2} \xi}$. The solution $l_{*}(\xi)$ can also be written as a power series using equation (A8),

$l_{*}(\xi)=\frac{c_{*}}{c_{2}}\left(\frac{\xi}{c_{2}}\right)^{(\beta-3) / 2}\left[(\beta-1) \sum_{n} \frac{t^{\beta-1+2 n}}{\beta-1+2 n}+\sum_{n} \frac{t^{\beta+1+2 n}(2 n+2)}{(\beta+2 n)(\beta+1+2 n)}\right]$

from which we can extract the asymptotic behaviour for vanishing $\xi$

$$
l_{*}(\xi) \simeq c_{*} \xi^{\beta-2} \text {. }
$$

These results imply $\Phi(x)$ has an exponentially vanishing $x \rightarrow \infty$ tail (see [9])

$$
\Phi(x) \simeq \frac{\pi^{2} x}{c_{2}^{2}} \mathrm{e}^{-\pi^{4} x /\left(4 c_{2}\right)}
$$

while $\Phi_{*}(x)$ has a power-law one

$$
\Phi_{*}(x) \simeq A x^{-(\beta-1)} .
$$

Therefore we may repeat the same consideration as in the surface probability calculation: even if $\Phi_{*}(x)$ is suppressed by powers of $r$, it is the most important term for large $v / r^{2}$.

\section{A.2. $2<\beta<3$}

In this case the expansion of $g_{r}(\lambda)$ reads

$$
g_{r}\left(\exp \left(-\frac{u}{r^{1 /(\beta-2)}}\right)\right)=1-\frac{a(u)}{r^{1 /(\beta-2)}}+\cdots
$$

with the conditions

$$
a(0)=0 \quad \text { and } \quad a^{\prime}(0)=1 .
$$


The surface probability distribution and its generating function $G_{r}^{s}(\lambda)$ can then be written as functions of scaled variables as

$$
\begin{aligned}
& \operatorname{Pr}\left[s_{r}(o)=s\right]=\frac{1}{r^{d_{\mathrm{c}}-1}} \phi\left(\frac{s}{r^{d_{\mathrm{c}}-1}}\right)+\cdots \\
& G_{r}^{s}\left(\exp \left(-u / r^{d_{\mathrm{c}}-1}\right)\right)=a^{\prime}(u)+\cdots
\end{aligned}
$$

where $\phi(x)$ is the inverse Laplace transform of $a^{\prime}(x)$. As before, by substituting equation (A9) in the recurrence equation for $g_{r}(\lambda)$ and by equating the numerators of the subleading terms, we obtain the differential equation

$$
u a^{\prime}(u)=a(u)-c_{*}(\beta-2) a(u)^{\beta-1} .
$$

The solution, using conditions (A10), reads

$$
a(u)=\frac{u}{\left[1+c_{*}(\beta-2) u^{\beta-2}\right]^{1 /(\beta-2)}} .
$$

Now, from the asymptotic behaviour of $a^{\prime}(u)$

$a^{\prime}(u)=\left[1+c_{*}(\beta-2) u^{\beta-2}\right]^{-(\beta-1) /(\beta-2)} \simeq \begin{cases}1-c_{*}(\beta-1) u^{\beta-2} & \text { for } u \rightarrow 0 \\ {\left[c_{*}(\beta-2)\right]^{-(\beta-1) /(\beta-2)} u^{-\beta+1}} & \text { for } u \rightarrow \infty\end{cases}$ we can determine that of the scaling function $\phi(x)$

$$
\phi(x) \sim \begin{cases}x^{-(\beta-1)} & \text { for } x \rightarrow \infty \\ x^{\beta-2} & \text { for } x \rightarrow 0\end{cases}
$$

which has the expected power-law behaviour.

Let us now turn to the volume probability. The conditions $h_{r}(1)=1$ and $h^{\prime}(r)=r$ lead to the expansion

$$
h_{r}\left(\exp \left(-\xi r^{-d_{\mathrm{c}}}\right)\right)=1-\frac{b(\xi)}{r^{d_{\mathrm{c}}-1}}+\cdots
$$

with

$$
b(0)=0 \quad b^{\prime}(0)=1 .
$$

The differential equation for $b(\xi)$ is found by substituting this expansion in the recurrence (16); it reads

$$
(\beta-1) \xi b^{\prime}(\xi)=b(\xi)-c_{*}(\beta-2) b(\xi)^{\beta-1}+(\beta-2) \xi
$$

By direct substitution one can easily verify that

$$
b(\xi)=\left(\xi / c_{*}\right)^{1 /(\beta-1)}
$$

is a solution, but does not satisfy the second condition of equation (A12). However, we obtain a separable differential equation if we let $b(\xi)=\xi^{1 /(\beta-1)} \bar{b}(\xi)$,

$$
\bar{b}^{\prime}(\xi)=\frac{\beta-2}{\beta-1}\left(1-c_{*} \bar{b}(\xi)^{\beta-1}\right) \xi^{-\frac{1}{\beta-1}} .
$$

The solution is now implicitly given by

$$
\begin{aligned}
& \bar{b}_{2} F_{1}\left(1, \frac{1}{\beta-1} ; \frac{\beta}{\beta-1} ; c_{*} \bar{b}^{\beta-1}\right)=\xi^{\frac{\beta-2}{\beta-1}} \\
& \sum_{k=0}^{\infty} \frac{1}{1+k(\beta-1)} c_{*}^{k} \bar{b}^{1+k(\beta-1)}=\xi^{\frac{\beta-2}{\beta-1}}
\end{aligned}
$$


This allows us to determine the asymptotic behaviour of the solution $b(\xi)$

$$
b(\xi) \sim \begin{cases}\xi-\left(c_{*} / \beta\right) \xi^{\beta-1} & \text { for } \xi \rightarrow 0 \\ \xi^{1 /(\beta-1)} & \text { for } \xi \rightarrow \infty\end{cases}
$$

The volume probability and its scaled generating function can now be written as

$$
\begin{aligned}
& G_{r}^{v}\left(\exp \left(-\xi / r^{d_{\mathrm{c}}}\right)\right)=l(\xi)+\cdots \\
& \operatorname{Pr}\left[v_{r}(o)=v\right]=\frac{1}{r^{d_{\mathrm{c}}}} \Phi\left(\frac{v}{r^{d_{\mathrm{c}}}}\right)+\cdots
\end{aligned}
$$

where $l(\xi)$ is the Laplace transform of $\Phi(x)$. As before $l(0)=1$ by normalization and $l(\xi)$ is obtained from the differential equation

$$
\xi l^{\prime}(\xi)=-c_{*}(\beta-2) l(\xi) b(\xi)^{\beta-2} .
$$

The small and large $\xi$ asymptotic behaviour of $l(\xi)$ can be obtained from that of $b(\xi)$, and read

$$
l(\xi) \simeq \begin{cases}1-c_{*} \xi^{\beta-2} & \text { for } \xi \rightarrow 0 \\ \exp \left(-c_{*}(\beta-1) \xi^{(\beta-2) /(\beta-1)}\right) & \text { for } \xi \rightarrow \infty .\end{cases}
$$

Therefore the asymptotic behaviour of $\Phi(x)$ reads

$$
\Phi(x) \sim \begin{cases}x^{-(\beta-1)} & \text { for } x \rightarrow \infty \\ x^{-\beta / 2} \exp \left(-k x^{2-\beta}\right) & \text { for } x \rightarrow 0\end{cases}
$$

with

$$
k=c_{*}^{\beta-1}(\beta-2)^{\beta-2} .
$$

The probability distribution is exponentially vanishing for small volumes and it has a powerlaw tail with exponent $1-\beta$, as expected.

\section{Appendix B. $V_{n}$ moments}

First of all, let us write the explicit form of $\mathcal{F}_{n}\left(V_{1}, V_{2}, \ldots, V_{n-1}\right)$,

$$
\mathcal{F}_{n}\left(V_{1}, V_{2}, \ldots, V_{n-1}\right)=\sum_{k=2}^{n} \sum_{n_{1}=1}^{n-1} \ldots \sum_{n_{k}=1}^{n-1} \delta\left(n-\sum_{j=1}^{k} n_{i}\right) \prod_{j=1}^{k} V_{n_{j}} .
$$

Now we can write an expression for $E^{V}\left[V_{n}\right]$ and its generating function $E^{\lambda}\left[V_{n}\right]$ involving only $V_{1}, V_{2}, \ldots, V_{n-1}$

$$
\begin{aligned}
E^{V}\left[V_{n}\right]=\sum_{z} & f_{z} \sum_{\left\{V_{k, j}\right\}} \delta\left(V_{1}-1-\sum_{j} V_{1, j}\right) \\
& \times \sum_{j}\left\{V_{n, j}+\mathcal{F}_{n}\left(V_{1, j}, V_{2, j}, \ldots, V_{n-1, j}\right)\right\} \prod_{j} P\left(\left\{V_{k, j}\right\}\right) \\
= & \sum_{z} f_{z} \sum_{V_{1, j} \delta}\left(V_{1}-1-\sum_{j} V_{1, j}\right) \\
& \times \sum_{j}\left\{E^{\left.V_{1, j}\left[V_{n}\right]+E^{V_{1, j}}\left[\mathcal{F}_{n}\left(V_{1}, V_{2}, \ldots, V_{n-1}\right)\right]\right\} \prod_{j^{\prime} \neq j} P\left(V_{1, j^{\prime}}\right)}\right.
\end{aligned}
$$




$$
\begin{aligned}
E^{\lambda}\left[V_{n}\right] & =\lambda \sum_{z} f_{z}(z-1)\left\{E^{\lambda}\left[V_{n}\right]+E^{\lambda}\left[\mathcal{F}_{n}\left(V_{1}, V_{2}, \ldots, V_{n-1}\right)\right]\right\} G(\lambda)^{z-2} \\
& =\lambda g^{\prime}(G(\lambda))\left\{E^{\lambda}\left[V_{n}\right]+E^{\lambda}\left[\mathcal{F}_{n}\left(V_{1}, V_{2}, \ldots, V_{n-1}\right)\right]\right\} \\
& =\left[1-\lambda g^{\prime}(G(\lambda))\right]^{-1} \lambda g^{\prime}(G(\lambda)) E^{\lambda}\left[\mathcal{F}_{n}\left(V_{1}, V_{2}, \ldots, V_{n-1}\right)\right]
\end{aligned}
$$

This shows that $E^{\lambda}\left[V_{n}\right]$ is more singular than $E^{\lambda}\left[\mathcal{F}_{n}\left(V_{1}, V_{2}, \ldots, V_{n-1}\right)\right]$, which has now to be calculated. To do this we need an expression for the generic multiple moment $E^{\lambda}\left[V_{1}^{k_{1}} V_{2}^{k_{2}} \cdots V_{n}^{k_{n}}\right]$. First of all, note that powers of $V_{1}$ can be extracted, that is

$$
E^{V}\left[V_{1}^{k_{1}} V_{2}^{k_{2}} \cdots V_{n}^{k_{n}}\right]=V^{k_{1}} E^{V}\left[V_{2}^{k_{2}} \cdots V_{n}^{k_{n}}\right]
$$

The recurrence rule for $E^{\lambda}\left[V_{2}^{k_{2}} \cdots V_{n}^{k_{n}}\right]$ reads

$$
\begin{aligned}
E^{\lambda}\left[V_{2}^{k_{2}} \cdots V_{n}^{k_{n}}\right] & =\left[1-\lambda g^{\prime}(G(\lambda))\right]^{-1}\left\{\lambda g^{\prime}(G(\lambda)) \sum_{j=2}^{n} E^{\lambda}\left[V_{2}^{k_{2}} \ldots k_{j} V_{j}^{k_{j}-1} \mathcal{F}_{j} \cdots V_{n}^{k_{n}}\right]\right. \\
& +\lambda g^{\prime \prime}(G(\lambda)) \sum_{k_{j, 1}, k_{j, 2}} E^{\lambda}\left[V_{2}^{k_{2,1}} \cdots V_{n}^{k_{n, 1}}\right] E^{\lambda}\left[V_{2}^{k_{2,2}} \cdots V_{n}^{k_{n, 2}}\right]+\cdots \\
& \left.+\lambda g^{(N)}(G(\lambda)) \sum_{k_{j, 1}, \ldots, k_{j, N}} \prod_{l=1}^{N} E^{\lambda}\left[V_{2}^{k_{2, l}} \cdots V_{n}^{k_{n, l}}\right]\right\}
\end{aligned}
$$

where $N=\sum_{j} k_{j}$, the sums over $k_{j, l}$ are such that $\sum_{l} k_{j, l}=k_{j}$, and only most singular terms are kept. Equation (B1) can be recursively used; the moments on the right-hand side involve either products with fewer terms or lower $j V_{j}$ so that we end with moments of $V_{1}$, which are trivial.

The general expression of $E^{\lambda}\left[V_{2}^{k_{2}} \cdots V_{n}^{k_{n}}\right]$ for both $2<\beta<3$ and $\beta>3$ cases

$$
E^{\lambda}\left[V_{1}^{k_{1}} V_{2}^{k_{2}} \cdots V_{n}^{k_{n}}\right] \simeq \begin{cases}(1-\lambda)^{\left[1-\sum_{j} k_{j}((2 \beta-3) j-\beta+2)\right] /(\beta-1)} & 2<\beta<3 \\ (1-\lambda)^{\frac{1}{2}-\frac{1}{2} \sum_{j} k_{j}(3 j-1)} & \beta>3\end{cases}
$$

can now be proved by induction. Substituting equation (B2) on the right-hand side of equation (B1), we see that all terms have the same singular behaviour when $2<\beta<3$, while the $g^{\prime}(G(\lambda))$ and $g^{\prime \prime}(G(\lambda))$ terms are the leading order ones for $\beta>3$.

The branch probability moments just calculated are related to the tree probability ones by

$$
\begin{aligned}
\widehat{E}^{\lambda}\left[V_{n}\right] & =\lambda \widehat{g}^{\prime}(G(\lambda))\left[E^{\lambda}\left[V_{n}\right]+E^{\lambda}\left[\mathcal{F}_{n}\left(V_{1}, V_{2}, \ldots, V_{n-1}\right)\right]\right] \\
& =\lambda \widehat{g}^{\prime}(G(\lambda)) E^{\lambda}\left[V_{n}\right]+\cdots
\end{aligned}
$$

and

$$
\widehat{E}^{\lambda}\left[V_{2}^{k_{2}} \cdots V_{n}^{k_{n}}\right]=\lambda \widehat{g}^{\prime}(G(\lambda)) E^{\lambda}\left[V_{2}^{k_{2}} \cdots V_{n}^{k_{n}}\right]+\cdots .
$$

Therefore $\widehat{E}^{\lambda}\left[V_{1}^{k_{1}} \cdots V_{n}^{k_{n}}\right]$ has the same asymptotic behaviour (equation (B2)) as $E^{\lambda}\left[V_{1}^{k_{1}} \cdots V_{n}^{k_{n}}\right]$. This implies the following asymptotic behaviour of $\widehat{E}^{V}\left[V_{1}^{k_{1}} V_{2}^{k_{2}} \cdots V_{n}^{k_{n}}\right]$

and finally

$$
\widehat{E}^{V}\left[V_{1}^{k_{1}} V_{2}^{k_{2}} \cdots V_{n}^{k_{n}}\right] \simeq \begin{cases}V^{\left[\sum_{j} k_{j}((2 \beta-3) j-\beta+2)-\beta\right] /(\beta-1)} & 2<\beta<3 \\ V^{\frac{1}{2} \sum_{j} k_{j}(3 j-1)-\frac{3}{2}} & \beta>3\end{cases}
$$

$$
\left\langle V_{1}^{k_{1}} V_{2}^{k_{2}} \cdots V_{n}^{k_{n}}\right\rangle_{V} \simeq \begin{cases}V^{\sum_{j} k_{j}((2 \beta-3) j-\beta+2) /(\beta-1)} & 2<\beta<3 \\ V^{\frac{1}{2} \sum_{j} k_{j}(3 j-1)} & \beta>3 .\end{cases}
$$




\section{References}

[1] Newman M E J 2002 Preprint cond-mat/0202208

Albert R and Barabasi A-L 2002 Rev. Mod. Phys. 7447

[2] Stauffer D and Aharony A 1994 Introduction to Percolation Theory (London: Taylor \& Francis)

[3] Ambjorn J, Durhuus B and Jonsson T 1990 Phys. Lett. B 244403

Ambjorn J, Durhuus B and Jonsson T 1997 Quantum Geometry (Cambridge: Cambridge University Press)

[4] Bollobas B 1985 Random Graphs (New York: Academic)

[5] Barabasi A-L and Albert R 1999 Science 286509

[6] Burda Z, Erdmann J, Petersson B and Wattenberg M 2003 Phys. Rev. E 67026105

[7] Burda Z, Correia J D and Krzywicki A 2001 Phys. Rev. E 64046118

[8] Havlin S and Ben-Avraham D 1987 Adv. Phys. 36 695-798

[9] Destri C and Donetti L 2002 J. Phys. A: Math. Gen. 355147

[10] Suzuki M 1983 Prog. Theor. Phys. 6965

[11] Alexander S and Orbach R 1982 J. Physique Lett. 43625

[12] Burioni R and Cassi D 1995 Phys. Rev. E 512865

Cassi D and Regina S 1992 Mod. Phys. Lett. B 61397

[13] Hattori K, Hattori T and Watanabe H 1987 Prog. Theor. Phys. Suppl. 92108

[14] Athreya K B and Ney P E 1972 Branching Processes (New York: Springer)

[15] Kesten H 1987 Ann. Inst. H Poincaré Probab. Stat. 22425

[16] Destri C and Donetti L 2002 J. Phys. A: Math. Gen. 359499 